CORRIGENDUM

doi:10.1038/nature13610

\title{
Corrigendum: Proviral silencing in embryonic stem cells requires the histone methyltransferase ESET
}

Toshiyuki Matsui, Danny Leung, Hiroki Miyashita, Irina A. Maksakova, Hitoshi Miyachi, Hiroshi Kimura, Makoto Tachibana, Matthew C. Lorincz \& Yoichi Shinkai

Nature 464, 927-931 (2010); doi:10.1038/nature08858

In Fig. 1a of this Letter, it has come to our attention that the lanes for the Dnmt3l ${ }^{+/+}$and $D n m t 3 l^{-/-}$testes RNA samples were run on different gels (northern blots) but were not displayed as such. The Dnmt3l ${ }^{-1-}$ sample was originally intended as a positive control for derepression of different retroelements analysed in this paper, such as LINE-1 and IAP. However, owing to space limitations, these samples were only mentioned in the legend to Fig. 1a. Regardless, according to Nature's publication guidelines, these lanes should have been displayed separately. In addition, we have now inserted a black line between lanes 4 and 5 (counting from the right-hand side) of Fig. $2 \mathrm{a}$ and lanes 1 and 2 (counting from the right-hand side) of Supplementary Fig. 3a because although they were run on the same gel, they were not adjacent in the original gel. The Supplementary Information of this Corrigendum shows the corrected Supplementary Fig. 3a and the raw data used to prepare Figs 1a and $2 \mathrm{a}$ and Supplementary Fig. 3a. These corrections do not change our interpretation of the results or the conclusions drawn in this Letter. Nevertheless, we apologize for any confusion the original display may have caused.

Supplementary Information is available in the online version of this Corrigendum.
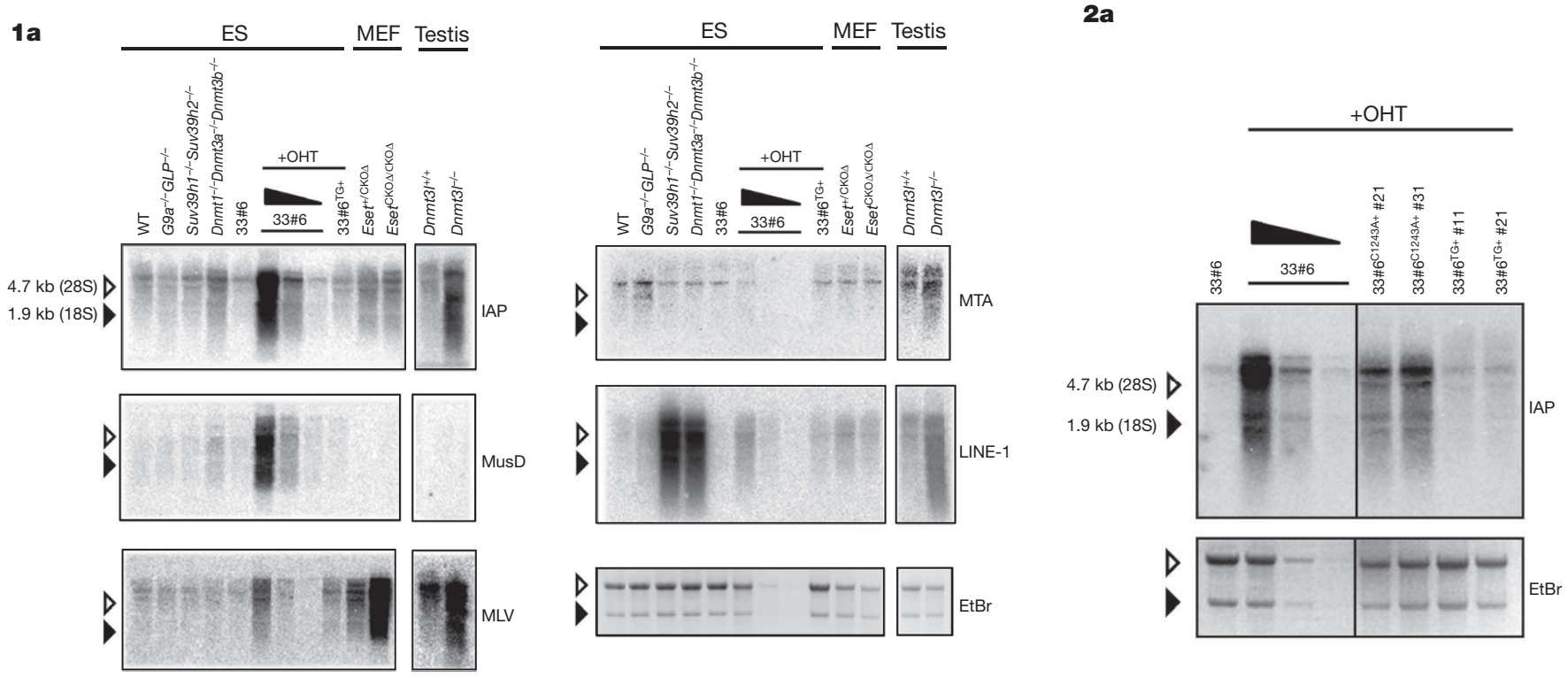

Figure $1 \mid$ This figure shows the corrected Figs 1a and 2a of the original Letter. 